\title{
EXTENDING A JORDAN RING HOMOMORPHISM
}

\author{
ROBERT LEWAND
}

\begin{abstract}
In this paper a homomorphism from an ideal $\mathfrak{B}$ of a quadratic Jordan algebra $\mathfrak{I}$ without 2-torsion over a ring $\Phi$ onto a unital quadratic Jordan algebra $\mathfrak{I}^{\prime}$ without 2-torsion is extended to a homomorphism from $\mathfrak{I}$ to $\mathfrak{J}^{\prime}$. We then show if $D$ is any class of quadratic Jordan algebras without 2-torsion, then the upper radical property determined by $D$ is hereditary.
\end{abstract}

1. Preliminaries. We adopt the notation and terminology of an earlier paper [3] concerning quadratic Jordan algebras and hereditary radical properties. For a discussion of upper radical properties the reader is referred to [1] and [2]. Basically, given a class of rings $D$ with the property that any nonzero ideal of a ring of $D$ can be mapped homomorphically onto a ring of $D$, then $D$ can be extended to a class of all $D$ semisimple rings: a ring is $D$ radical if it cannot be mapped homomorphically onto a ring of $D$; a ring without $D$ radical ideals is $D$ semisimple.

\section{Two theorems.}

THEOREM 1. Given a quadratic Jordan algebra $\mathfrak{I}$ with ideal $\mathfrak{B}$ and a unital quadratic Jordan algebra $\mathfrak{I}^{\prime}$ without 2-torsion, a homomorphism $\varphi$ from $\mathfrak{B}$ onto $\mathfrak{I}^{\prime}$ can be extended to a homomorphism $\tilde{\varphi}: \mathfrak{I} \rightarrow \mathfrak{I}$.

Proof. Since $\varphi$ is an onto homomorphism, some element of $\mathfrak{B}$, say $b$, maps into 1 . Define $\tilde{\varphi}: \mathfrak{J} \rightarrow \mathfrak{I}^{\prime}$ by $\tilde{\varphi}(a)=\varphi\left(U_{b} a\right)$. $\tilde{\varphi}$ is clearly linear. For $b^{\prime} \in \mathfrak{B}$,

$$
\tilde{\varphi}\left(b^{\prime}\right)=\varphi\left(U_{b} b^{\prime}\right)=U_{\varphi(b)} \varphi\left(b^{\prime}\right)=U_{1} \varphi\left(b^{\prime}\right)=\varphi\left(b^{\prime}\right) .
$$

It is therefore established that $\tilde{\varphi}$ extends $\varphi$. It now remains to show that $\tilde{\varphi}$ is indeed a homomorphism. Since $2 U_{x}=V_{x}^{2}-V_{x^{2}}$, under the assumption that $\mathfrak{I}^{\prime}$ has no 2-torsion, it is sufficient to show that $\tilde{\varphi}$ preserves the $V$ operator, i.e. to show $\tilde{\varphi}\left(a \circ a^{\prime}\right)=\tilde{\varphi}(a) \circ \tilde{\varphi}\left(a^{\prime}\right)$ for $a, a^{\prime} \in \mathfrak{I}$. For then $\tilde{\varphi}\left(2 U_{x} y\right)=2 U_{\tilde{\varphi}(x)} \tilde{\varphi}(y)$ and $2\left\{\tilde{\varphi}\left(U_{x} y\right)-U_{\tilde{\varphi}(x)} \tilde{\varphi}(y)\right\}=0$ implies $\tilde{\varphi}\left(U_{x} y\right)-$ $U_{\tilde{\varphi}(x)} \tilde{\varphi}(y)=0$. First a simplification: it will be convenient to express $U_{b} U_{b} V_{a} a^{\prime}$ as

$$
U_{b}\left[(b \circ a) \circ\left(b \circ a^{\prime}\right)\right]-\left(U_{b} a\right) \circ\left(U_{b} a^{\prime}\right)-b \circ\left\{b a U_{b} a^{\prime}\right\},
$$

Received by the editors November 16, 1972.

AMS (MOS) subject classifications (1970). Primary 17C30.

Key words and phrases. Quadratic Iordan algebra, radical property.

(c) American Mathematical Society 1973 
an identity which is easily verified in the case of special Jordan algebras and is therefore true for all Jordan algebras as a consequence of Macdonald's theorem [4]. Now, since $\tilde{\varphi}\left(b^{\prime}\right)=\varphi\left(b^{\prime}\right)=\varphi\left(U_{b} b^{\prime}\right)$ for $b^{\prime} \in \mathfrak{B}$,

$$
\begin{aligned}
\tilde{\varphi}\left(a \circ a^{\prime}\right) & =\varphi\left(U_{b}\left(a \circ a^{\prime}\right)\right)=\varphi\left(U_{b} U_{b}\left(a \circ a^{\prime}\right)\right) \\
& =\varphi\left(U_{b}\left[(b \circ a) \circ\left(b \circ a^{\prime}\right)\right]-\left(U_{b} a\right) \circ\left(U_{b} a^{\prime}\right)-b \circ\left\{b a U_{b} a^{\prime}\right\}\right) .
\end{aligned}
$$

Consider these terms one at a time.

$$
\begin{aligned}
& \varphi\left(U_{b}\left[(b \circ a) \circ\left(b \circ a^{\prime}\right)\right]\right)=\varphi\left(U_{b}\left[\left(V_{b} a\right) \circ\left(V_{b} a^{\prime}\right)\right]\right)=\varphi\left[\left(V_{b} a\right) \circ\left(V_{b} a^{\prime}\right)\right] \\
& \quad=\varphi\left(V_{b} a\right) \circ \varphi\left(V_{b} a^{\prime}\right)=\varphi\left(U_{b} V_{b} a\right) \circ \varphi\left(U_{b} V_{b} a^{\prime}\right)=\varphi\left(V_{b} U_{b} a\right) \circ \varphi\left(V_{b} U_{b} a^{\prime}\right) \\
& \quad=V_{1} \varphi\left(U_{b} a\right) \circ V_{1} \varphi\left(U_{b} a^{\prime}\right)=2 \tilde{\varphi}(a) \circ 2 \tilde{\varphi}\left(a^{\prime}\right)=4\left(\tilde{\varphi}(a) \circ \tilde{\varphi}\left(a^{\prime}\right)\right) .
\end{aligned}
$$

Next,

Finally,

$$
\varphi\left[\left(U_{b} a\right) \circ\left(U_{b} a^{\prime}\right)\right]=\varphi\left(U_{b} a\right) \circ \varphi\left(U_{b} a^{\prime}\right)=\tilde{\varphi}(a) \circ \tilde{\varphi}\left(a^{\prime}\right) .
$$

$$
\begin{gathered}
\varphi\left(b \circ\left\{b a U_{b} a^{\prime}\right\}\right)=\varphi\left(V_{b} V_{b, a} U_{b} a^{\prime}\right)=\varphi\left(V_{b} U_{b, U(b) a^{a}} a\right) \\
=\varphi\left[U_{b, U(b) a}\left(b \circ a^{\prime}\right)+U_{b, U(b) a^{\prime}}(b \circ a)-2\left(U_{b} a\right) \circ\left(U_{b} a^{\prime}\right)\right] \\
\quad \text { (by Macdonald's theorem) } \\
=\varphi\left[U_{b, U(b) a}\left(b \circ a^{\prime}\right)\right]+\varphi\left[U_{b, U(b) a^{\prime}}(b \circ a)\right]-2\left[\varphi\left(U_{b} a\right) \circ \varphi\left(U_{b} a^{\prime}\right)\right] \\
=\varphi\left[U_{b, U(b) U(b) a}\left(b \circ a^{\prime}\right)\right]+\varphi\left[U_{b, U(b) U(b) a^{\prime}}(b \circ a)\right]-2\left[\tilde{\varphi}(a) \circ \tilde{\varphi}\left(a^{\prime}\right)\right] \\
=\varphi\left\{U_{b}\left[\left(U_{b} a\right) \circ\left(U_{b} a^{\prime}\right)\right]+\left(U_{b} a^{\prime}\right) \circ\left(U_{b} U_{b} a\right)\right\} \\
\quad+\varphi\left\{U_{b}\left[\left(U_{b} a\right) \circ\left(U_{b} a^{\prime}\right)\right]+\left(U_{b} a\right) \circ\left(U_{b} U_{b} a^{\prime}\right)\right\}-2\left(\tilde{\varphi}(a) \circ \tilde{\varphi}\left(a^{\prime}\right)\right) \\
\quad \text { (again using Macdonald's Theorem) } \\
=2\left(\tilde{\varphi}(a) \circ \tilde{\varphi}\left(a^{\prime}\right)\right) .
\end{gathered}
$$

Combining these results, $\tilde{\varphi}\left(a \circ a^{\prime}\right)=\tilde{\varphi}(a) \circ \tilde{\varphi}\left(a^{\prime}\right)$. $\tilde{\varphi}$ is therefore a homomorphism and the proof of the theorem is complete.

THEOREM 2. If $D$ is any class of quadratic Jordan rings with unit element over a ring $\Phi$ and no 2-torsion, satisfying the condition that any nonzero ideal of a ring of $D$ can be mapped homomorphically onto a ring of $D$, then the upper radical property determined by $D$ is hereditary.

Proof. Let $\mathfrak{I}$ be a $D$ radical ring and let $\mathfrak{B}$ be a nonzero ideal of $\mathfrak{J}$. Assume $\mathfrak{B}$ is not a $D$ radical ring. Then $\mathfrak{B}$ can be mapped homomorphically onto some ring $\mathfrak{S}$ in the class $D$. By the above theorem $\mathfrak{J}$ is also homomorphic to $\mathfrak{S}$ which contradicts $\mathfrak{I}$ being $D$ radical.

\section{REFERENCES}

1. T. Anderson, N. Divinsky and A. Sulinski, Hereditary radicals in associative and alternative rings, Canad. J. Math. 17 (1965), 594-603. MR 31 \#215. 
2. A. Kuroš, Radicals of rings and algebras, Mat. Sb. 33 (75) (1953), 13-26. (Russian) MR 15, 194.

3. R. Lewand, Hereditary radicals in Jordan rings, Proc. Amer. Math. Soc. 33 (1972), 302-306.

4. R. Lewand and K. McCrimmon, Macdonald's theorem for quadratic Jordan algebras, Pacific J. Math. 35 (1970), 681-706.

Department of Mathematics, Windham College, Putney, Vermont 05346 